\section{Nonimmediate Hypersensitivity Reaction to Rifaximin Confirmed With a Drug Challenge Test}

\author{
Moya B, García-Moguel I, Mielgo R, Herráez L, Crespo JF \\ Allergy Service, Hospital Universitario 12 de Octubre, Madrid, \\ Spain
}

J Investig Allergol Clin Immunol 2021; Vol. 31(5): 453-454 doi: $10.18176 /$ jiaci.0660

Key words: Drug challenge test. Drug hypersensitivity. Maculopapular exanthema. Nonimmediate reaction. Rifamycin. Rifaximin.

Palabras clave: Prueba de provocación con medicamentos. Hipersensibilidad a medicamentos. Exantema maculopapular. Reacción no inmediata. Rifamicina. Rifaximina.

Rifaximin is a broad-spectrum antibiotic that is increasingly used to treat gastrointestinal infections caused by a variety of enteric pathogens including gram-positive, gram-negative, aerobic, and anaerobic bacteria such as enterotoxigenic and enteroaggregative Escherichia coli, and Salmonella, Shigella, Campylobacter, Plesiomonas, and Aeromonas species [1,2]. It belongs to the rifamycin family, a group of antibiotics synthesized either artificially or naturally by the bacterium Amycolatopsis rifamycinica and that includes rifampicin (rifampin), rifabutin, rifapentine, rifalazil, and rifaximin [3]. Unlike other rifamycins, rifamixin contains an additional pyridoimidazole ring, which minimizes systemic absorption, thus leading to high gastrointestinal concentrations $[3,4]$. Given its excellent safety profile, minimal drug interactions, and negligible impact on the intestinal microbiome, rifaximin is increasingly used to treat intestinal infections, irritable bowel syndrome with small intestinal bacterial overgrowth, and hepatic encephalopathy in patients with chronic liver disease, as well as for treatment and prevention of uncomplicated diverticular disease $[4,5]$. Although rifaximin is increasingly used, hypersensitivity reactions are rare. To our knowledge, we report the first case of a nonimmediate hypersensitivity reaction to rifaximin confirmed with a drug challenge test (DCT).

The patient was a 67-year-old woman with a medical history of anxiety and depression, chronic diarrhea, irritable bowel syndrome with small bowel bacterial overgrowth, 2 previous episodes of acute diverticulitis, previous papillary thyroid carcinoma (treated with surgery and radioactive iodine), and mild asthma. She was referred to our allergy department for evaluation of an adverse event related to treatment with rifaximin. After being diagnosed with acute diverticulitis, the patient was prescribed a course of rifaximin $400 \mathrm{mg}$ every 8 hours. Three hours after the first dose, she experienced nausea, vomiting, and increasingly frequent diarrheic stools. Despite these symptoms, the patient continued rifaximin normally and, on day 4 , developed a maculopapular erythematous and pruritic rash over her thorax, abdomen, back, and extremities. She did not develop fever, blisters, pustules, mucosal involvement, skin hyperpigmentation, or desquamation. Her skin lesions resolved spontaneously 7 days after discontinuation of rifaximin.

After obtaining the patient's written informed consent, we performed an allergological work-up, including skin prick test (SPT) and a graded DCT with rifaximin. SPT was performed with rifaximin at $1 \mathrm{mg} / \mathrm{mL}$ on the volar aspect of the forearm and yielded a negative result [6]. On the same day, the patient underwent a DCT, consisting of a graded oral challenge starting at $50 \mathrm{mg}$ and followed by $150 \mathrm{mg}$ 30 minutes later, up to a final dose of $200 \mathrm{mg}$. One hour after the DCT, the patient felt nauseous, with diarrheic stool that resolved spontaneously without medication. The DCT was considered inconclusive and repeated 24 hours later. Three hours after completion of the second DCT, the patient again experienced diarrheic stools. Ten hours after the DCT, she developed a maculopapular erythematous and pruritic skin rash on her abdomen and left arm (Figure). She was treated with oral prednisone $0.5 \mathrm{mg} / \mathrm{kg} / \mathrm{d}$ for 3 days and loratadine $10 \mathrm{mg} / \mathrm{d}$ for 7 days until the skin lesions resolved, without desquamation or residual lesions.

The rationale for an allergological study with rifaximin was that the patient had a long history of gastrointestinal infections, in which rifaximin is an effective first-line therapy. Although reactions to rifamycins (eg, fever, rash, flu-like syndrome, acute renal failure, hemolytic anemia, thrombocytopenia, and anaphylaxis) have been reported [7], few reactions to rifaximin have been described since it was approved $[3,4]$. In the present case, the patient initially developed gastrointestinal symptoms 3 hours after receiving the first dose of rifaximin; these were considered a nonimmune adverse reaction to rifaximin. She continued treatment, developing a mild maculopapular

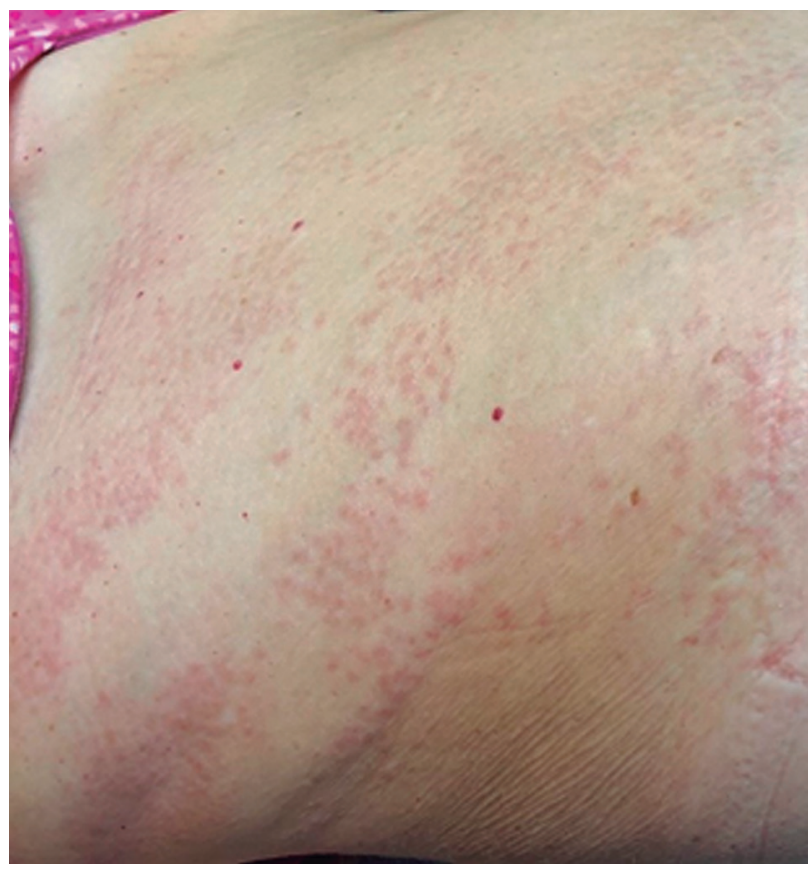

Figure. Maculopapular erythematous and pruritic rash on the abdomen during drug challenge testing with rifaximin. 
rash on day 4 with no life-threatening signs, suggesting a hypersensitivity reaction. However, as the patient continued rifaximin through day 4 , the drug latency period could not be determined. Consequently, the allergological study began with an SPT instead of patch tests because the underlying immunological mechanism of the reaction was initially unclear. The graded rifaximin challenge elicited cutaneous symptoms compatible with the case history, although an IgEmediated mechanism could not be demonstrated with skin testing. Antonicelli et al [8] reported the case of a 64-year-old man with IgE-mediated allergies to rifaximin and rifamycin SV who experienced 2 episodes of anaphylaxis, one after oral administration of rifaximin and the second after topical rifamycin SV. Serum-specific IgE to rifampicin, rifabutin, and rifapentine was positive. Patel et al [4] reported the case of a 62-year-old woman admitted to hospital with an episode of hepatic encephalopathy and possible toxic epidermal necrolysis (TEN) to rifaximin, although she received other possible culprit drugs during admission and a skin biopsy could not be performed to confirm the diagnosis [4]. Fritz et al [9] reported the case of a 42-year-old woman with Stevens-Johnson syndrome (SJS)/TEN confirmed with skin biopsy, most likely due to rifaximin. However, the patient also received other possible culprit drugs, such as spironolactone. No allergology work-up to determine the culprit drug was performed. In this case, skin lesions improved after the patient discontinued both rifaximin and spironolactone. Yang et al [10] aimed to identify possible culprit drugs for SJS and TEN in a nationwide database in South Korea, finding only 1 suspected case with rifaximin.

Although rifaximin is widely prescribed for certain gastrointestinal infections, hypersensitivity reactions are rare. To our knowledge, we present the first case of a nonimmediate hypersensitivity reaction to rifaximin confirmed by DCT where an IgE-mediated mechanism could not be demonstrated with an allergology study. Given that rifaximin shares a structure with other rifamycins, patients diagnosed with immediate or nonimmediate allergy to this drug should be recommended to avoid the rifamycin family, unless allergy is ruled out by specific tests.

\section{Funding}

The authors declare that no funding was received for the present study.

\section{Conflicts of Interest}

The authors declare that they have no conflicts of interest.

\section{References}

1. Koo HL, DuPont HL. Rifaximin: a unique gastrointestinalselective antibiotic for enteric diseases. Curr Opin Gastroenterol. 2010;26(1):17-25.

2. Gomi H, Jiang ZD, Adachi JA, Ashley D, Lowe B, Verenkar $M P$, et al. In vitro antimicrobial susceptibility testing of bacterial enteropathogens causing traveler's diarrhea in four geographic regions. Antimicrob Agents Chemother. 2001Jan;45(1):212-6.

3. Lin SW, Lin CJ, Yang JC. Rifamycin SV MMX for the treatment of traveler's diarrhea. Expert Opin Pharmacother. 2017;18(12):1269-77.

4. Patel AS, Supan EM, Ali SN. Toxic epidermal necrolysis associated with rifaximin. Am J Health Syst Pharm. 2013;70(10):874-6.

5. Shayto RH, Abou Mrad R, Sharara Al. Use of rifaximin in gastrointestinal and liver diseases. World J Gastroenterol. 2016;22(29):6638-51.

6. García F, Blanco J, Carretero P, Herrero D, Juste S, Garcés M, et al. Anaphylactic reactions to topical rifamycin. Allergy. 1999 May;54(5):527-8.

7. Martinez E, Collazos J, Mayo J. Hypersensitivity reactions to rifampin. Pathogenetic mechanisms, clinical manifestations, management strategies, and review of the anaphylactic-like reactions. Medicine (Baltimore). 1999;78:361-9.

8. Antonicelli L, Micucci C, Bilò MB, Manfredi M, Valentini $M$, Campi P. IgE-mediated reactions to rifaximin and rifamycin SV and cross-reactivity among rifamycins. Allergy. 2009;64(8):1232-3.

9. Fritz CD, Adebajo C, Aronsohn A, Jensen DM. A New-Onset Rash in the Setting of Rifaximin Treatment for Hepatic Encephalopathy. ACG Case Rep J. 2014;2(1):42-4.

10. Yang MS, Lee JY, Kim J, Kim GW, Kim BK, Kim JY, et al. Searching for the Culprit Drugs for Stevens-Johnson Syndrome and Toxic Epidermal Necrolysis from a Nationwide Claim Database in Korea. J Allergy Clin Immunol Pract. 2020 Feb;8(2):690-5.e2

Manuscript received September 8, 2020; accepted for publication December 14, 2020.

Beatriz Moya

E-mail: drbeatrizmoya@gmail.com 